\title{
Claudin-1 expression in cervical cancer
}

\author{
FRIEDERIKE HOELLEN ${ }^{1}$, ANNIKA WALDMANN ${ }^{2}$, CONSTANZE BANZ-JANSEN ${ }^{3}$, UWE HOLTRICH ${ }^{4}$, \\ THOMAS KARN ${ }^{4}$, MARTINA OBERLÄNDER $^{5,6}$, JENS K. HABERMANN ${ }^{5,6}$, MAREIKE HÖRMANN $^{7}$, \\ FRANK KÖSTER $^{1}$, JULIKA RIBBAT-IDEL ${ }^{8}$, MARC THILL ${ }^{9}$, ACHIM RODY $^{1}$, \\ AHMED EL-BALAT $^{4^{*}}$ and LARS HANKER ${ }^{1 *}$
}

\begin{abstract}
${ }^{1}$ Department of Gynecology and Obstetrics, and ${ }^{2}$ Institute for Social Medicine and Epidemiology, University of Lübeck, D-23538 Lübeck; ${ }^{3}$ Department of Gynecology and Obstetrics, University Hospital Oldenburg, D-26133 Oldenburg; ${ }^{4}$ Department of Obstetrics and Gynecology, Goethe-University, D-60590 Frankfurt; ${ }^{5}$ Section for Translational Surgical Oncology and Biobanking, Department of Surgery; ${ }^{6}$ Interdisciplinary Center for Biobanking-Lübeck, University of Lübeck and University Medical Center Schleswig-Holstein, Campus Lübeck, D-23538 Lübeck; ${ }^{7}$ Institute for Pathology, University of Düsseldorf, D-40225 Düsseldorf; ${ }^{8}$ Institute for Pathology, University of Lübeck, D-23538 Lübeck;

${ }^{9}$ Department of Gynecology and Obstetrics, Agaplesion Markus Krankenhaus, D-60431 Frankfurt, Germany
\end{abstract}

Received March 10,2017; Accepted August 17, 2017

DOI: $10.3892 / \mathrm{mco} .2017 .1391$

\begin{abstract}
Claudin-1 is a tight junction protein that has been demonstrated to be involved in tumorigenesis and tumor progression in various types of solid tumors. In the present study, the protein expression of claudin-1 in squamous cervical cancer tissues obtained from 106 patients was analyzed by immunohistochemistry. In addition, the grade of claudin-1 expression was analyzed for associations with certain clinicopathological parameters. A significant overexpression of claudin-1 was detected in the tumor cells, when compared with that in the peritumoral stroma. There was no significant association between claudin-1 expression and FIGO stage, tumor size, grading or the appearance of distant metastases. Cervical cancer patients scoring positive for claudin- 1 protein expression tended to exhibit more lymph node metastasis (28.3\%), compared with claudin-1-negative patients (7.1\%). Regarding overall survival, the results of the present study suggest a better prognosis for claudin-1-negative patients. In order to elucidate whether claudin-1 overexpression has a significant prognostic impact on squamous cervical cancer, further studies are required.
\end{abstract}

Correspondence to: Dr Friederike Hoellen, Department of Gynecology and Obstetrics, University of Lübeck, Ratzeburger Allee 160, D-23538 Lübeck, Germany

E-mail: friederike.hoellen@uksh.de

*Contributed equally

Key words: cervical cancer, claudin-1, squamous cervical cancer, immunohistochemistry, lymph node metastases, survival analysis

\section{Introduction}

The tight junction protein claudin-1 is an integral part of the epithelial and endothelial tight junction complex, which executes signal transduction pathways and cellular transport functions $(1,2)$. A total of 24 tissue-specific claudin subtypes have been identified (3). Loss of tight junction complex integrity serves a role in tumorigenesis of solid tumors, and it has been hypothesized that claudin-1 can act tissue-specifically as a tumor suppressor or as an oncoprotein (4). Thus, claudin-1 can be either downregulated or upregulated according to the specific tumor entity $(2,5)$. Claudins are crucial for cell migration, invasion and metastasis; this was demonstrated in a xenograft colorectal cancer mouse model, where tumor growth and metastasis were linked to E-Cadherin expression and $\beta$-catenin/T-cell factor (TCF) signaling cascade (5). In basal-like high-grade ductal breast cancer, colorectal cancer, gastric cancer, melanoma and cervical cancer, the protein expression of claudin-1 was enhanced, whereas in hepatocellular carcinoma, esophageal cancer and prostate cancer, claudin-1 expression was reduced (5). Initial attempts have been made to introduce pre-clinical tests for an anti-claudin antibody-based anti-tumor-therapy targeting the second extracellular domain of claudin- 3 and -4 which is overexpressed in pancreatic, prostate, breast and ovarian cancer. The antibodies could carry radionuclides, toxins or the Clostridium perfringens enterotoxin (CPE). CPE binding to the extracellular domain of claudin-3 or -4 interferes with the membrane-pore complex and causes cell lysis via the disruption of the cellular osmotic equilibrium (2).

In cervical cancer claudin-1 expression is elevated according to the stage of invasiveness. In vitro studies have demonstrated that claudin-1 is involved in the tumorigenesis of cervical cancer $(3,6,7)$. In cervical intraepithelial neoplasia, claudin-1 expression levels correlate with $\mathrm{p} 16^{\mathrm{INK} 4 \mathrm{a}}$, increasing with the severity of cervical dysplasia (8). However, a correlation of claudin-1 expression with certain clinicopathological 
parameters, and a possible prognostic value for claudin-1 in cervical cancer, has yet to be demonstrated. In the present study, claudin-1 expression in cervical cancer tissues was analyzed and cross-referenced with clinicopathological data in order to elucidate the impact of claudin-1 on cervical cancer prognosis.

\section{Patients and methods}

Patients. Tissue samples were obtained from the resected tumors of 106 patients with early squamous cervical cancer, collected consecutively at the University Medical Center Schleswig-Holstein, Campus Lübeck (Lübeck, Germany) between January 2003 and November 2011. The patients (mean age, 52.86 years; range, 25-79 years) underwent a radical hysterectomy and $\geq 1$ lymph node was removed for the detection of tumor cells. Patients who received neoadjuvant therapies were excluded from the study prior to sample collection. The Ethics Committee of the University of Lübeck approved the study protocol. All patients provided written informed consent to allow retrospective data analysis on the basis of an anonymized dataset.

Histochemical analysis. Details of the histopathological diagnosis, FIGO stage, patient characteristics and adjuvant therapies were retrieved from hospital records, along with follow-up data. Cervical cancer tissue and corresponding peritumoral stromal tissue samples were used to construct tissue microarrays (TMAs), as described previously (9). Biopsies were procured from paraffinized tissue blocks and cores were inserted into a standard-sized recipient array block. The TMAs were constructed using a semi-automated arrayer (TMArrayer ${ }^{\mathrm{TM}}$; Pathology Devices, Inc., Westminster, MD, USA). Four replicate cores were obtained from each sample. In order to include all samples, three TMA blocks were constructed.TMA sections $(2 \mu \mathrm{m})$ were mounted on glass slides, dewaxed in xylene and rehydrated through graded ethanol to water. Antigens were retrieved by microwaving the sections in $10 \mathrm{mM}$ citrate buffer ( $\mathrm{pH}$ 6.0) for $20 \mathrm{~min}$ at $800 \mathrm{~W}$. Blocking was performed using antibody dilution buffer (DCS Innovative Diagnostik-Systeme; Dr. Christian Sartori GmbH \& Co., KG, Hamburg, Germany) at room temperature for $15 \mathrm{~min}$. The rabbit polyclonal claudin-1 antibody (cat. no. RB-9209; Thermo Fisher Scientific, Inc., Waltham, MA, USA) was diluted 1:100 in the same buffer and incubated with the sections for $60 \mathrm{~min}$ at room temperature. For the negative control, the primary antibody was replaced with PBS. A goat anti-rabbit secondary antibody (cat. no. DAB-088153; dilution, 1:2,500; Dianova $\mathrm{GmbH}$, Hamburg, Germany) was subsequently used for $1 \mathrm{~h}$ at room temperature. For the detection of immunoreactivity, the Dako REAL ${ }^{\text {TM }}$ Detection System Alkaline Phosphatase/RED (Dako; Agilent Technologies GmbH, Waldbronn, Germany) was used, according to the manufacturer's protocol. Then, the sections were counterstained with Hematoxylin Solution, Gill No. 3 (Sigma-Aldrich; Merck KGaA, Darmstadt, Germany). IHC was assessed using a Leica DM5000B light microscope (Leica Microsystems GmbH, Wetzlar, Germany) by a pathologist in a blinded manner according to the immunoreactivity score (IRS) established by Remmele and Stegner (10). The IRS combines a score for staining intensity from 0-3 ( 0 , no color reaction; 1 , mild; 2 , moderate; 3 , intense) multiplied with the score for the percentage of positive cells from $0-4(0$, no positive cells; $1,<10 \% ; 2,10-50 \% ; 3,51-80 \% ; 4,>80 \%)$. The combination results in the IRS, ranging from $0-12$.

Statistical analysis. Statistical analysis was conducted using SPSS software version 22 (IBM Corp., Armonk, NY, USA) and graphs were created in Microsoft Excel 2013 (Microsoft Corporation, Redmond, WA, USA). Continuous data are presented as the means \pm standard deviation. The patients' age at diagnosis is reported together with the age-range. Categorical and nominal data are presented as absolute and relative frequencies. The Student's independent samples t-test, Mann-Whitney U-test and Kruskal-Wallis test, were used to identify differences of claudin-1 expression between subgroups defined by clinicopathological characteristics. The Chi-squared and Fisher's exact tests were used to evaluate differences regarding molecular markers and clinicopathological characteristics between patients with and without claudin-1 expression. The differences in prognosis between cervical cancer patients with and without claudin-1 expression were analyzed using the log-rank test, and Kaplan-Meier survival curves were plotted. $\mathrm{P}<0.05$ was considered to indicate a statistically significant result.

\section{Results}

In the patient cohort, the protein expression of claudin-1 was significantly higher in cervical cancer tissues, compared with in the peritumoral environment $(\mathrm{P}<0.001$; Mann-Whitney U-test). In 104/106 (98\%) cases, staining for claudin-1 was not detected in cervical tissue of the peritumoral environment (IRS=0). In cervical tumor tissues claudin-1 was overexpressed to differing extents in 92/106 cases (87\%; Figs. 1 and 2). Overall, 49 cervical cancer tissue samples exhibited a maximum claudin-1 expression of $\mathrm{IRS}=12$.

No correlation was observed between claudin-1 expression levels and tumor size (T), lymphovascular space invasion $(\mathrm{L})$, distant metastasis $(\mathrm{M})$, grade $(\mathrm{G})$, vessel invasion $(\mathrm{V})$ or FIGO stage (Table I). Regarding lymph node metastasis (N), claudin-1 positive cervical cancer tissue samples (IRS=1-12) had an increased frequency of lymph node involvement in comparison with claudin-1 negative cervical cancer tissues (IRS=0; 7.1 vs. 28.3\%; Table II). However, this observation was not significant ( $\mathrm{P}=0.110$; two-tailed Fisher's Exact test), which may be partly due to the small amount of claudin-1 negative cancer cases among all 106 samples $(n=14)$.

Claudin-1 expression was not significantly associated with p53, epidermal growth factor receptor, the proliferation-associated antigen detected with the antibody Molecular Immunology Borstel-1 (MIB-1) or CD-3 either in cancer tissues or in the peritumoral stroma. However, a positive correlation was observed between claudin-1 and p16 expression in cancer tissues $(\mathrm{P}=0.002$; Kruskal-Wallis test; Fig. 3$)$.

Follow-up data from $\leq 10$ years was available for 98 patients; all were included in the survival analysis. The overall survival curve indicated a worse prognosis for claudin-1-positive patients, as compared with for claudin-1-negative patients (Fig. 4). However, this difference was not determined to be statistically significant. 
Table I. Clinical and pathological parameters and expression of claudin-1 (associations not significant).

\begin{tabular}{|c|c|c|c|}
\hline Patients & $\begin{array}{c}\text { Total } \\
\text { (106 patients) } \\
\mathrm{n}(\%)\end{array}$ & $\begin{array}{c}\text { Claudin-1 negative } \\
\text { (IRS, } 0 ; \mathrm{n}=14) \\
\mathrm{n}(\%)\end{array}$ & $\begin{array}{c}\text { Claudin-1 positive } \\
\text { (IRS } \geq 1 ;=92) \\
\mathrm{n}(\%)\end{array}$ \\
\hline Age, mean $\pm \mathrm{SD}$ (range) & $52.86 \pm 12.48(25-79)$ & $50 \pm 13.96(25-74)$ & $53.3 \pm 12.26(26-79)$ \\
\hline \multicolumn{4}{|l|}{ Type } \\
\hline T0 & $2(1.9)$ & 0 & $2(2.2)$ \\
\hline $\mathrm{T} 1$ & $64(61)$ & $10(76.9)$ & $54(58.7)$ \\
\hline $\mathrm{T} 2$ & $35(33.3)$ & $3(23.1)$ & $32(34.8)$ \\
\hline $\mathrm{T} 3$ & $2(1.9)$ & 0 & $2(2.2)$ \\
\hline $\mathrm{T} 4$ & $2(1.9)$ & 0 & $2(2.2)$ \\
\hline $\mathrm{N}$ & - & & \\
\hline No & $79(74.5)$ & $13(92.9)$ & $66(71.7)$ \\
\hline $\mathrm{N} \leq 1$ & $27(25.5)$ & $1(7.1)$ & $26(28.3)$ \\
\hline \multicolumn{4}{|l|}{ M } \\
\hline M0 & $102(96.2)$ & $13(92.9)$ & 89 (96.7) \\
\hline $\mathrm{M} \leq 1$ & $4(3.8)$ & $1(7.1)$ & $3(3.3)$ \\
\hline \multicolumn{4}{|l|}{ Hemangiosis } \\
\hline Negative & $48(98)$ & $4(100)$ & $44(97.8)$ \\
\hline Positive & $1(2)$ & 0 & $1(2.2)$ \\
\hline Unknown & 57 & & - \\
\hline \multicolumn{4}{|l|}{ Lymphangiosis } \\
\hline Negative & $40(67.8)$ & $4(66.7)$ & $36(67.9)$ \\
\hline Positive & $19(32.2)$ & $2(33.3)$ & $17(32.1)$ \\
\hline Unknown & 47 & & \\
\hline \multicolumn{4}{|l|}{ Grading } \\
\hline G1 & $2(1.9)$ & 0 & $2(2.2)$ \\
\hline $\mathrm{G} 2$ & $48(46.6)$ & $3(23.1)$ & $45(50)$ \\
\hline G3 & $53(51.5)$ & $10(76.9)$ & $43(47.8)$ \\
\hline Gx & 3 & & \\
\hline \multicolumn{4}{|l|}{ FIGO } \\
\hline FIGO I & $64(60.4)$ & $10(71.4)$ & $54(58.7)$ \\
\hline FIGO II & $36(34)$ & $4(28.6)$ & $32(34.8)$ \\
\hline FIGO III & $4(3.8)$ & 0 & $4(4.3)$ \\
\hline FIGO IV & $2(1.9)$ & 0 & $2(2.2)$ \\
\hline \multicolumn{4}{|l|}{ Smoking habits } \\
\hline Smokers & $46(43.4)$ & $6(42.9)$ & $40(43.5)$ \\
\hline
\end{tabular}

SD, standard deviation; T, tumor size; N, lymph node metastasis; M, metastasis.

Table II. Claudin-1 expression in IRS and lymph node metastases (no significant correlation).

\begin{tabular}{lccc}
\hline & $\begin{array}{c}\text { Claudin-1 } \\
\text { negative } \\
\text { (IRS=0) } \\
(\%)\end{array}$ & $\begin{array}{c}\text { Claudin-1 } \\
\text { positive } \\
(\text { IRS=1-12) } \\
\text { stage }(\mathrm{n}, \%)\end{array}$ & $\begin{array}{c}\text { Total } \\
(\%)\end{array}$ \\
\hline N0 & $13(92.9)$ & $66(71.7)$ & $79(74.5)$ \\
N1 & $1(7.1)$ & $26(28.3)$ & $27(25.5)$ \\
\hline
\end{tabular}

N0, no lymph node metastases; N1, lymph node metastases; IRS, immunoreactivity score.

\section{Discussion}

A significantly higher expression level of claudin-1 was identified in squamous cervical cancer tissues obtained from the patient collective, as compared with in the peritumoral stroma, as determined by immunohistochemistry. This observation is concordant with the results of prior studies $(3,11)$. Dysregulation of claudin-1 has previously been demonstrated to be involved in tumorigenesis and progression in various malignant entities, including cervical cancer $(2,5)$. The pivotal role of claudin- 1 in tumorigenesis has been hypothesized in the context of the pre-cancerous 


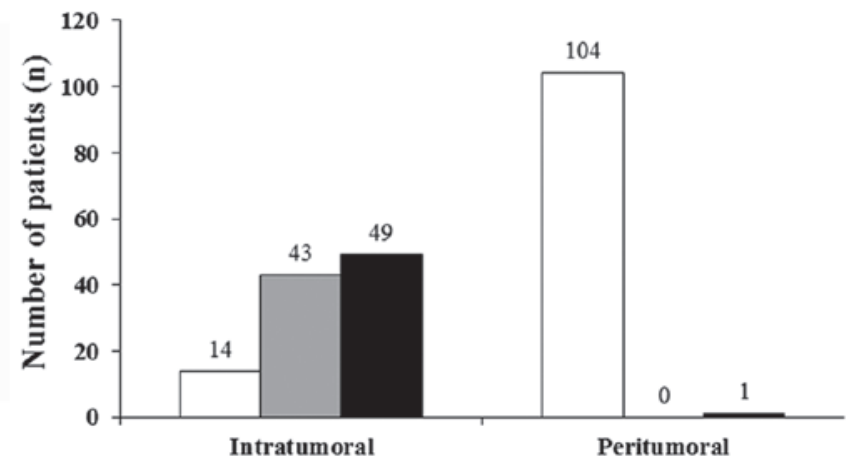

Figure 1. Claudin-1 expression according to the immunoreactivity score (Remmele and Stegner).

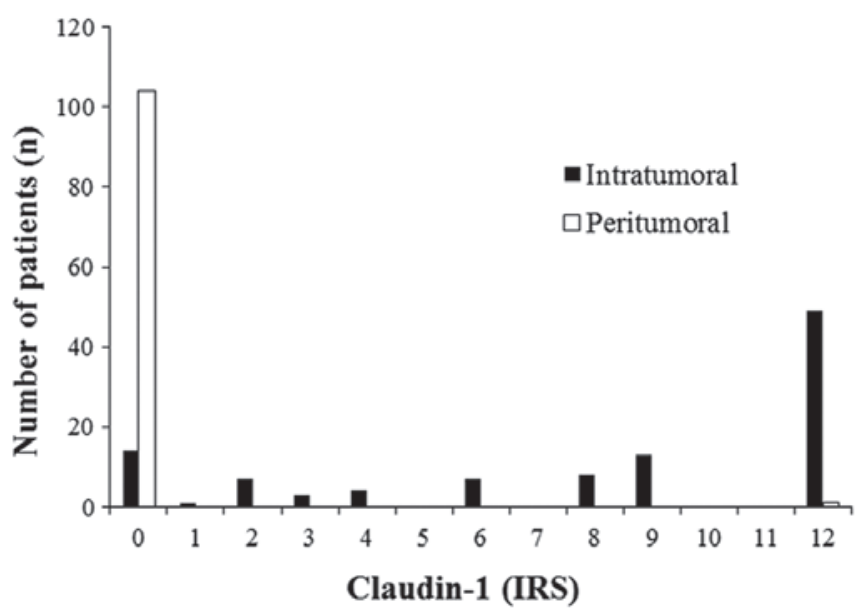

Figure 2. Intratumoral and peritumoral Claudin-1 scores (immunoreactivity score).

cervical lesions, intraepithelial neoplasia (3). Certain studies have proposed claudin-1 to be a tumor marker or a marker of pre-cancerous lesions equivalent to $\mathrm{p} 16^{\mathrm{INK} 4 \mathrm{a}}(8)$. However, the findings of the current study did not determine any significant association between the claudin-1 expression levels in the cervical cancer patient cohort and the tumor size, FIGO stage or grade. Thus, it is hypothesized that claudin-1 overexpression may be an early event in cervical cancer tumorigenesis, and that each specific cervical cancer tissue is defined by a characteristic grade of claudin-1 expression. Notably, higher levels of claudin-1 expression were identified in patients with lymph node metastases (not statistically significant). Further studies with larger patient cohorts are necessary to elucidate whether claudin-1 serves a role in lymph node metastasis. A recent publication by Zhang et al (11) corroborated this observation: The authors revealed correlation between claudin-1 overexpression in cervical cancer and lymph node metastasis $(\mathrm{P}<0.05)$. However, Zhang et al (11) analyzed a relatively small patient collective (73 patients). In contrast to the present study, the authors used an IHC scoring system based on the percentage of stained cells. Furthermore, the authors opted to use cervical tissues obtained from healthy females as the controls. In the current study, peritumoral

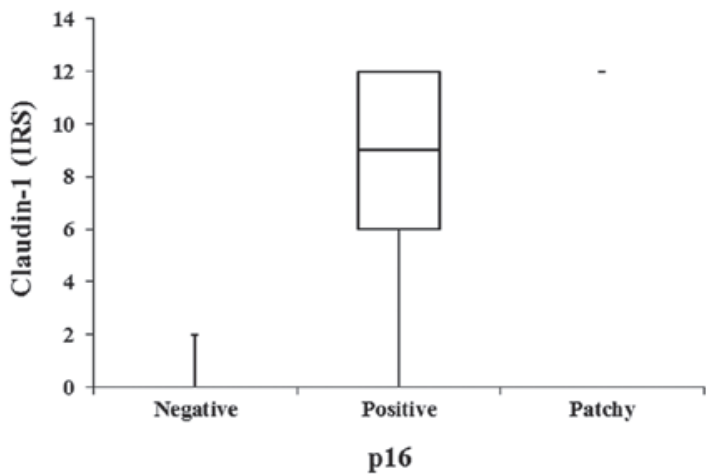

Figure 3. Intratumoral claudin-1 and p16 expression.

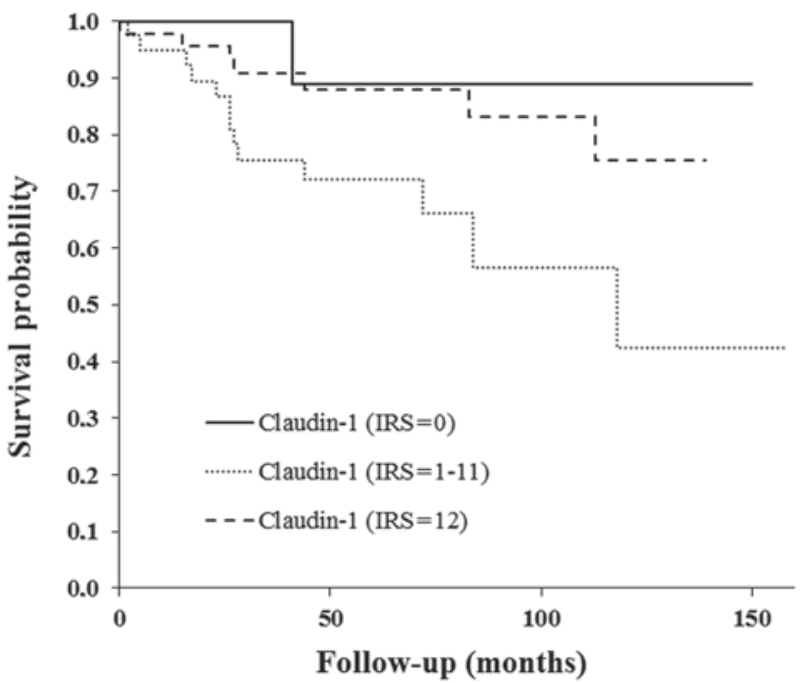

Figure 4. Overall survival according to the expression of claudin-1 (immunoreactivity score).

cervical tissues were used as the controls in order to exclude other possible confounders that may interfere with claudin-1 expression (e.g. immunologic mechanisms due to infection). IRS scoring according to Remmele and Stegner (10) offers an IHC scoring system that considers the quantity as well as the quality of cell staining. Furthermore, a significant correlation was demonstrated between claudin-1 expression and p16 expression in cervical cancer tissues, data that has not yet been published. However, one study with $>350$ patients reported a correlation between $\mathrm{p} 16^{\mathrm{INK} 4 \mathrm{a}}$ and claudin-1 expression in pre-invasive cervical lesions (8). Consequently claudin- 1 could serve as a marker for pre-invasive lesions and early invasive cervical carcinoma. Further studies are required to investigate the course of claudin-1 expression in pre-invasive lesions and consecutive invasive lesions.

The overall survival curve indicated a worse prognosis for claudin-1-positive patients when compared with claudin-1-negative patients (Fig. 4). However, this observation is not significant, which could be attributed to the small number of patients involved who had claudin-1-negative tumors $(\mathrm{n}=14$ with IRS $=0)$. Only one of these patients succumbed to the disease within the follow-up time. In the current patient 
collective, those with moderate claudin-1 expression had the worst prognosis. These data must be interpreted with care due to the small patient cohort, and further studies are required to clarify whether claudin-1 expression has a prognostic impact on cervical cancer. Zhang et al (11) also reported on the anti-apoptotic and invasive impact of claudin-1 in $\mathrm{SiHa}$ cells via the loss of E-cadherin and increased vimentin (11). Claudin-1 is a promising molecular marker in squamous cervical cancer. Its potential as a diagnostic, prognostic and therapeutic marker must be analyzed in further in vitro and in vivo studies.

\section{References}

1. Cunniffe C, Ryan F, Lambkin H and Brankin B: Expression of tight and adherens junction proteins in cervical neoplasia. Br J Biomed Sci 69: 147-153, 2012.

2. Kominsky SL: Claudins: Emerging targets for cancer therapy Expert Rev Mol Med 8: 1-11, 2006.

3. Szabó I, Kiss A, Schaff Z and Sobel G: Claudins as diagnostic and prognostic markers in gynecological cancer. Histol Histopathol 24: 1607-1615, 2009.

4. Swisshelm K, Macek R and Kubbies M: Role of claudins in tumorigenesis. Adv Drug Deliv Rev 57: 919-928, 2005.

5. Kwon MJ: Emerging roles of claudins in human cancer. Int J Mol Sci 14: 18148-18180, 2013.

6. Cunniffe C, Brankin B, Lambkin H and Ryan F: The role of claudin-1 and claudin-7 in cervical tumorigenesis. Anticancer Res 34: 2851-2857, 2014

7. Lee JW, Lee SJ, Seo J, Song SY, Ahn G, Park CS, Lee JH, Kim BG and Bae DS: Increased expressions of claudin-1 and claudin-7 during the progression of cervical neoplasia. Gynecol Oncol 97: 53-59, 2005.
8. Benczik M, Galamb Á, Koiss R, Kovács A, Járay B, Székely T, Szekerczés T, Schaff Z, Sobel G and Jeney C: Claudin-1 as a biomarker of cervical cytology and histology. Pathol Oncol Res 22: 179-188, 2016

9. Oberländer M, Alkemade H, Bünger S, Ernst F, Thorns C, Braunschweig T and Habermann JK: A 'waterfall' transfer-based workflow for improved quality of tissue microarray construction and processing in breast cancer research. Pathol Oncol Res 20: 719-726, 2014.

10. Remmele W and Stegner HE: Recommendation for uniform definition of an immunoreactive score (IRS) for immunohistochemical estrogen receptor detection (ER-ICA) in breast cancer tissue. Pathologe 8: 138-140, 1987 (In German).

11. Zhang WN, Li W, Wang XL, Hu Z, Zhu D, Ding WC, Liu D, Li KZ, Ma D and Wang H: CLDN1 expression in cervical cancer cells is related to tumor invasion and metastasis. Oncotarget 7: 87449-87461, 2016. 